\title{
Visualization of multimodal polymer-shelled contrast agents using ultrasound contrast sequences: an experimental study in a tissue mimicking flow phantom
}

\author{
Malin Larsson ${ }^{1 *}$, Matilda Larsson ${ }^{1,2}$, Letizia Oddo ${ }^{3}$, Silvia Margheritelli³ , Gaio Paradossi ${ }^{3}$, Jacek Nowak ${ }^{4}$,
} Lars-Åke Brodin ${ }^{1}$, Kenneth Caidahl ${ }^{2}$ and Anna Bjällmark ${ }^{1,2}$

\begin{abstract}
Background: A multimodal polymer-shelled contrast agent (CA) with target specific potential was recently developed and tested for its acoustic properties in a single element transducer setup. Since the developed polymeric CA has different chemical composition than the commercially available CAs, there is an interest to study its acoustic response when using clinical ultrasound systems. The aim of this study was therefore to investigate the acoustic response by studying the visualization capability and shadowing effect of three polymer-shelled CAs when using optimized sequences for contrast imaging.
\end{abstract}

Methods: The acoustic response of three types of the multimodal CA was evaluated in a tissue mimicking flow phantom setup by measuring contrast to tissue ratio (CTR) and acoustic shadowing using five image sequences optimized for contrast imaging. The measurements were performed over a mechanical index (MI) range of 0.2-1.2 at three CA concentrations $\left(10^{6}, 10^{5}, 10^{4}\right.$ microbubbles $\left./ \mathrm{ml}\right)$.

Results: The CTR-values were found to vary with the applied contrast sequence, MI and CA. The highest CTR-values were obtained when a contrast sequence optimized for higher Ml imaging was used. At a CA concentration of $10^{6}$ microbubbles/ml, acoustic shadowing was observed for all contrast sequences and CAs.

Conclusions: The CAs showed the potential to enhance ultrasound images generated by available contrast sequences. A CA concentration of $10^{6} \mathrm{MBs} / \mathrm{ml}$ implies a non-linear relation between $\mathrm{MB}$ concentration and image intensity.

Keywords: Acoustic shadowing, Contrast agent, Contrast sequences, Contrast to tissue ratio, Flow phantom, Multimodal

\section{Background}

In 1968, intravenously injectable air microbubbles (MBs) were introduced as a contrast agent (CA) for use with echocardiography in order to enhance echo from relatively weak echogenic regions such as the vascular lumen [1]. However, the instability of these free gas MBs with a lifetime of only a few seconds limited their practical use and

\footnotetext{
* Correspondence: malinl@sth.kth.se

'Department of Medical Engineering, School of Technology and Health, KTH Royal Institute of Technology, Alfred Nobels Allé 10, 141 52, Huddinge, Stockholm, Sweden

Full list of author information is available at the end of the article
}

their relatively large size hindered their passage through the pulmonary capillary. In order to improve the MB stability, attempts have been made to encapsulate gas within a thin shell and in 1984, the first MBs with ability to pass through the pulmonary circulation were developed [2]. However, the thin shell of these MBs still allowed air to diffuse across the shell structure. In order to optimize the MB stability, a low soluble gas, instead of air, was encapsulated within the shell and the gas leakage was thus limited. At present, CAs consisting of MBs with the improved stability are widely used during standard ultrasound

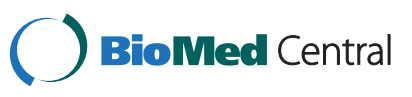


examinations, enabling e.g. tissue perfusion studies and endocardial border delineation $[3,4]$.

Although the commercially available CAs are relatively stable and well functioning in different clinical applications, there is still a need for improvement and extended applicability, such as targeted imaging and fusion imaging. In fact, polymer-shelled CAs with multimodality potential are currently under development $[5,6]$. The multimodality approach has the potential to increase the clinical value of contrast imaging by providing the possibility of retrieving anatomical and functional information from two or more imaging systems simultaneously. In addition, the attachment of specific antibodies and ligands to the shell surface could enable specific attraction of the MBs to intended targets. The CAs might also be employed as a carrier of drugs that could be released locally at chosen target sites by disruption of the MBs with high-energy ultrasound. Other advantages of these CAs are a long shelf life as well as a narrow size distribution that increases the image sensitivity, which is of primary importance during targeting imaging [7-9].

Nevertheless, it is important to remember that polymershelled MBs demonstrate a different acoustical behavior than lipid-shelled MBs due to differences in compressibility and visco-elastic properties. The acoustic properties of the polymeric CAs has so far been studied in-vitro using single element transducers (Poehlmann et al:: On the interplay of structural, mechanical and acoustic behaviour of multifunctional magnetic microbubbles, submitted) [10-11] and by using a commercially ultrasound scanner with in-house developed contrast sequences [12,13]. However, no in-vitro studies using available clinical ultrasound systems with optimized sequences for contrast imaging has been performed hitherto. Keeping this in mind, the aim of this study was to determine the acoustic response by studying the visualization capability of three polymer-shelled CAs - one plain ultrasound and two surface-modified MBs for combined ultrasound and magnetic resonance imaging - in a tissue mimicking flow phantom using different ultrasound contrast sequences. In addition, the acoustic shadowing at different concentrations of the developed CAs was studied.

\section{Material and methods}

An experimental setup including a tissue mimicking flow phantom, a reservoir tank and a peristaltic pump was designed as shown in Figure 1. The contrast to tissue ratio (CTR) and the acoustic shadowing were studied for three polymer-shelled CAs, using different ultrasound systems with associated contrast sequences. The tests were performed at three CA concentrations. For the CTRmeasurements, the commercially available CA SonoVue was used as reference.

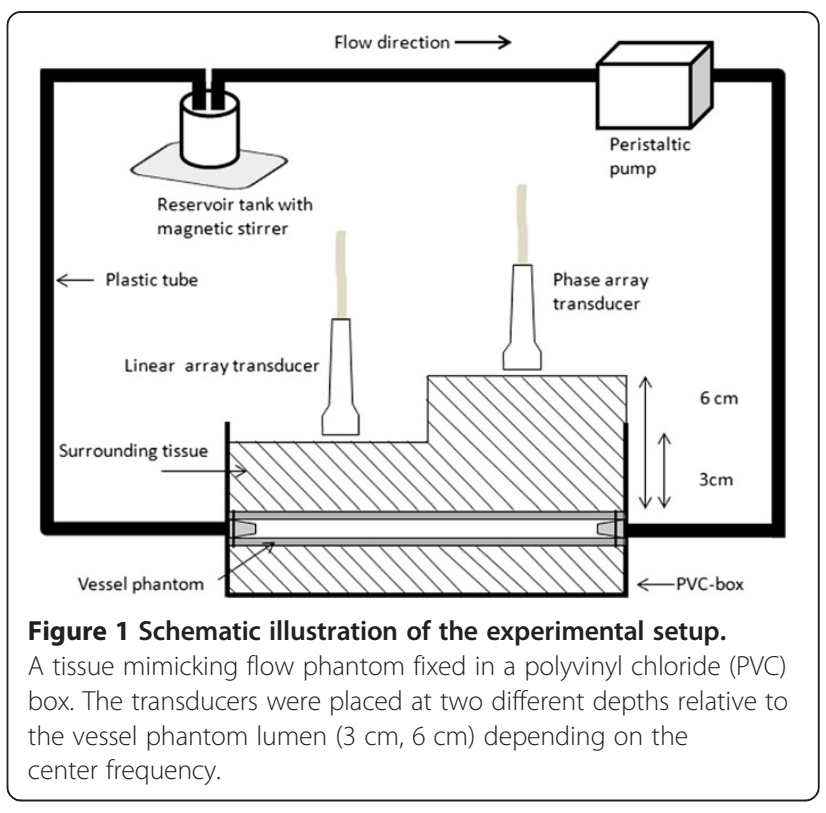

\section{Phantom design}

A tissue mimicking flow phantom with a vessel size of the human carotid artery was designed, see Figure 2. The vessel phantom was constructed by heating a mixture of $15 \%(\mathrm{w} / \mathrm{w})$ polyvinyl alcohol (PVA) (Sigma-Aldrich, St. Louise, MO, US), 3\% (w/w) graphite powder (particle size $<50 \mu \mathrm{m}$, Merck KGaA, Darmstadt, Germany) and deionized water to $90^{\circ} \mathrm{C}$. Graphite powder was added as acoustic scatters [14]. The mixture was then poured into a cylindrical vessel mould of acrylic plastic (diameter $12 \mathrm{~mm}$, length $100 \mathrm{~mm}$ ) and the vessel lumen was created by inserting a metal rod (diameter $6 \mathrm{~mm}$ ) into the vessel mould. Thereafter, it was stored in a freezer at $-20^{\circ} \mathrm{C}$ for $12 \mathrm{~h}$. During the subsequent $12 \mathrm{~h}$, the vessel mould was stored in room temperature. In order to obtain acoustical properties similar to tissue, the vessel phantom underwent three freeze-thaw cycles [15].

After the three freeze-thaw cycles, the vessel phantom was attached to plastic connectors on each side of a polyvinyl chloride (PVC) box $(100 \mathrm{~mm} \times 60 \mathrm{~mm})$, see Figure 1 .
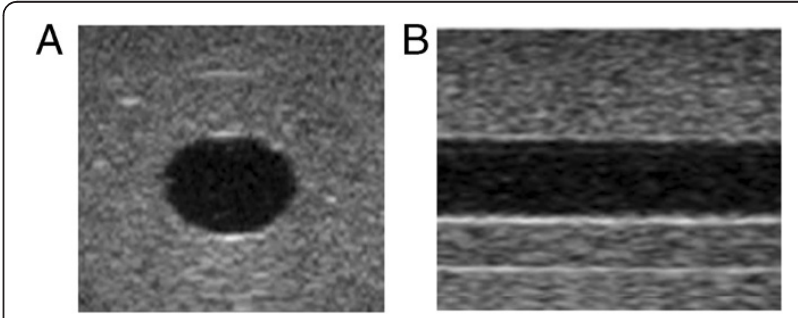

Figure 2 Ultrasound B-mode images of the flow phantom. Images acquired in the short axis (A) and the long axis view (B) Circulated solution consisted of de-ionized water. 
The inner part of the PVC-box was covered with a $4 \mathrm{~mm}$ thick rubber layer that acted as an ultrasound absorbing material. A surrounding tissue mimicking mixture of 3\% (w/w) agar (Merck KGaA, Darmstadt, Germany), 4\% $(\mathrm{w} / \mathrm{w})$ graphite powder (particle size $<50 \mu \mathrm{m}$, Merck KGaA, Darmstadt, Germany) and de-ionized water was heated to $85^{\circ} \mathrm{C}$. To prevent the vessel phantom from dissolving, the tissue mimicking mixture was cooled in room temperature to approximately $50^{\circ} \mathrm{C}$ before pouring it into the PVC-box. In addition, the vessel phantom lumen was filled with de-ionized water to prevent it from collapsing when pouring the tissue mimicking mixture into the PVC-box.

\section{Contrast agents}

Three in-house made polymer-shelled CAs were employed in the study (Table 1). One type was a plain polymershelled CA (Plain PVA) useful solely with ultrasound imaging [6]. The other two types had a modified shell or shell surface, both containing superparamagnetic iron oxide nanoparticles (SPIONs) for combined ultrasound and magnetic resonance imaging. However, the binding technique of the SPIONs differed. In the modified CA Type A (PVA Type A), the SPIONs were covalently linked to the shell surface through chitosan molecules, whereas in the modified CA Type B (PVA Type B), the SPIONs were embedded in the shell $[5,16]$. In addition, the commercially available lipid-shelled CA SonoVue ${ }^{\curvearrowleft}$ was used as a reference [17].

The stock solution concentrations of each CA were determined using a light microscope (Olympus, Hamburg, Germany) and a counting chamber (Marienfeld Superior, Lauda-Königshofen, Germany). The mean value of the number of MBs within 16 squares of the chamber, each with a volume of $6.25 \times 10^{-3} \mathrm{~mm}^{3}$, was calculated and displayed as the stock solution concentration. Subsequently, each stock solution was diluted with de-ionized water to desired concentration. In total, three concentrations of each type of CA were prepared, i. e. $10^{4}, 10^{5}$ and $10^{6} \mathrm{MBs} / \mathrm{ml}$.

\section{Experimental procedure}

The tissue mimicking flow phantom was connected to a peristaltic pump (Watson Marlow, Falmouth, United Kingdom) generating a pulsatile flow with a mean

Table 1 Properties of the contrast agents

\begin{tabular}{lll}
\hline Type of CA & Shell material & Gas composition \\
\hline Plain PVA & Polymer & Air \\
\hline PVA Type A & Polymer + SPION & Air \\
\hline PVA Type B & Polymer + SPION & Air \\
\hline SonoVue & Phospholipid & Sulfur hexafluoride \\
\hline
\end{tabular}

PVA = polyvinyl alcohol, $\mathrm{CA}=$ contrast agent, $\mathrm{SPION}=$ superparamagnetic iron oxide nanoparticles. velocity of $8.8 \mathrm{~cm} / \mathrm{s}$. The $500 \mathrm{ml}$ solution circulating in the closed system consisted of de-ionized water and CA, which underwent constant stirring in the reservoir tank to obtain an even distribution of CA in the solution. A cleaning procedure was performed after each experiment with a specific $\mathrm{CA}$ at a given concentration in order to remove all $\mathrm{MBs}$ in the closed system. During the cleaning procedure, the vessel phantom and the plastic tubes were washed with de-ionized water. In total, three cleaning procedures were performed before a new batch of CA was added to the system.

The employed ultrasound transducers were fixed by a tripod holder at two different distances from the vessel phantom lumen depending on the applied frequency. The low frequency transducers (M3S (GE Healthcare, Wisconsin, US) and S5-1 (Philips Healthcare, Amsterdam, The Netherlands)) were fixed at a distance of $6 \mathrm{~cm}$ from the vessel lumen while the high frequency transducers (M12L (GE Healthcare, Wisconsin, US) and 9 L4 (Siemens Healthcare, Erlangen, Germany)) were fixed $3 \mathrm{~cm}$ from the vessel lumen.

\section{Contrast sequences}

Four commonly used contrast sequences were employed in the study; pulse inversion (PI), power modulation (PM), contrast pulse sequence (CPS) and power pulse inversion (PPI) (Table 2). The common feature for all contrast sequences used, was the detection of the nonlinear response from the studied CA upon exposure to acoustic pressure. A brief description of each contrast sequences follows. In PI every second transmitted pulse was inverted compared to the previous, while in PM two pulses were transmitted in phase but with amplitude modifications [18]. For CPS a combination of both phase and amplitude modification was applied [19]. When applying PPI, a sequence of three pulses was transmitted for each echo line. The first and the third transmitted pulses are identical while the second transmitted pulse is an inverted replica of them [20].

\section{Data acquisition}

Ultrasound short-axis images $(\mathrm{n}=38)$ were acquired for four different contrast sequences (PI, PM, PPI and CPS) and three concentrations for each $\mathrm{CA}$ at a mechanical index (MI) ranging from 0.2 to maximal MI-value (0.8-1.2) of each ultrasound system tested (Table 2). A wide MI-range was used since the nonlinear responses from the CAs vary with applied acoustic pressure and the visco-elastic properties of the CA [21]. Before image acquisition, the focus point was placed in the middle of the vessel phantom lumen. The order of the image acquisition with the different contrast sequences was determined by a random number generator in Matlab $^{\oplus}$ (MathWorks, Natick, US) to minimize the influence of possible 
Table 2 Characteristics and settings of the contrast sequences

\begin{tabular}{llllll}
\hline Ultrasound system & Contrast sequence & Transducer & Frequency $(\mathbf{M H z})$ & Frame rate $(\mathbf{H z})$ & MI-range \\
\hline Vivid7, GE & PI & M12L & $5 / 10$ & 32.7 & $0.2-1.2$ \\
\hline Vivid7, GE & PI & M3S & $1.5 / 3.1$ & 25.7 & $0.2-0.8$ \\
\hline iE33, Philips & PM & S5-1 & $1.5 / 3.2$ & 39 & $0.2-1.2$ \\
\hline iE33, Philips & PPI & S5-1 & $1.3 / 2.6$ & 39 & $0.2-1.0$ \\
\hline Acuson sequoia 512, Siemens & CPS & 9 L4 & $4 / 8$ & 17 & $0.2-1.0$ \\
\hline
\end{tabular}

$\mathrm{PI}=$ pulse inversion, $\mathrm{PM}=$ power modulation, $\mathrm{PPI}=$ power pulse inversion, $\mathrm{CPS}=$ contrast pulse subtraction, $\mathrm{MI}=$ mechanical index .

concentration decrease of the CAs due to ultrasound exposure.

\section{Data analysis}

All the image sequences were analyzed offline using a specific work station for each ultrasound system; EchoPAC (GE Healthcare, Wisconsin, US), QLAB (Philips Healthcare Amsterdam, The Netherlands), Syngo (Siemens Healthcare, Erlangen, Germany).

\section{Contrast to tissue ratio}

Two regions of interest (ROIs) with an area of approximately $12.5 \mathrm{~mm}^{2}$ were manually placed in the middle of the vessel phantom lumen $\left(\mathrm{ROI}_{\text {Vessel }}\right)$ and in the surrounding tissue surrogate $\left(\mathrm{ROI}_{\mathrm{Tissue}}\right)$ at the same depth for all stored imaging sequences (Figure 3A). The ratio between the mean intensity (I), measured in decibel $(\mathrm{dB})$, within the $\mathrm{ROI}_{\text {Vessel }}$ and the $\mathrm{ROI}_{\text {Tissue }}$ was calculated and displayed as CTR (Equation 1).

$$
C T R=I_{R O I_{\text {Vessel }}}-I_{R O I_{\text {Tissue }}}
$$

\section{Acoustic shadowing}

In order to assess the acoustic shadowing, two identical ROIs, with an area of approximately $3 \mathrm{~mm}^{2}$, were manually placed in the upper and the lower part of the vessel

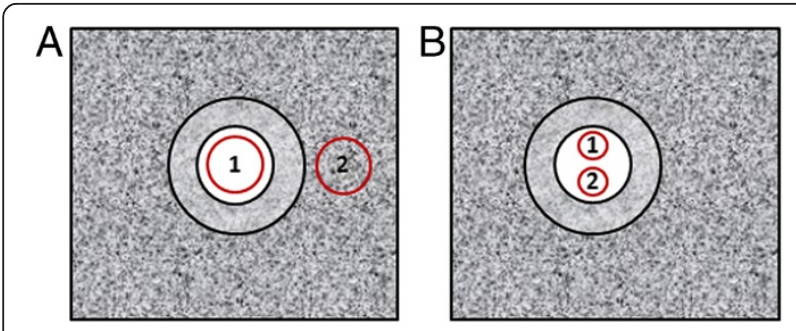

Figure 3 Off-line measurements. A) Illustration of the contrast to tissue ratio (CTR) measurements in the short axis view of the flow phantom. Two identical regions of interest (ROIs) were placed at the same depth in the short axis view; $\mathrm{ROI}_{\text {Vessel }}$ (1) and $\mathrm{ROI}_{\text {Tissue }}(2)$, B) Illustration of the acoustic shadowing measurements with two identical ROls placed underneath each other in the phantom vessel lumen; $\mathrm{ROI}_{\text {upper }}(1)$ and $\mathrm{ROI}_{\text {Iower }}(2)$. phantom lumen (Figure 3B) [22]. A shadowing effect was assumed if a significant higher intensity was observed for the upper ROI compared with the lower ROI. This was tested for each contrast sequence and CA concentration at the MI at which the maximal CTR-value was obtained using one-sided paired t-test (confidence interval of 95\%).

\section{Results}

\section{Contrast to tissue ratio}

The distribution of the CTR-values expressed as a function of MI for different CAs at a concentration of $10^{5}$ $\mathrm{MBs} / \mathrm{ml}$ obtained with different contrast sequences are presented in Figure 4. As can be seen, using the PI and PM sequences, the highest CTR-values were obtained at a lower MI (0.4), whereas with PPI sequence, the highest CTR-values appeared at higher MI (1.2). On the other hand, CPS sequence tended to produce the highest CTR-values at intermediate to higher MI $(0.6-1.0)$. A similar distribution pattern of the CTR-values was found for the two other CA concentrations $\left(10^{4}\right.$ and $10^{6} \mathrm{MBs} / \mathrm{ml}$ ) that were also tested (data not shown). This is further elucidated in Figure 5 where it shows the maximum CTR-values for each studied CA at a concentration of $10^{5} \mathrm{MBs} / \mathrm{ml}$ and the corresponding MI obtained with each contrast sequence employed. As can be seen from the figure, the PPI sequence provides the overall best performance, with the highest CTR-values for all studied CAs. A high dependency of the highest CTR values produced with different contrast sequences on the applied MI was also apparent in this study, a higher MI being optimal for use with PPI and CPS and a lower MI optimal for use with PI and PM sequences. Furthermore, PI and PM provided higher CTR-values for SonoVue ${ }^{\bullet}$ compared with the polymer-shelled CAs.

Figure 6 shows examples of ultrasound images of the tissue mimicking flow phantom containing Plain PVA at a concentration of $10^{5} \mathrm{MBs} / \mathrm{ml}$ obtained with different contrast sequences at maximum CTR-values. The figure illustrates in further detail the high CTR produced by PPI sequence (Figure 6C) as well as a clear frequencydependent difference in the images generated by PI with a high frequency transducer $(f=5 / 10 \mathrm{MHz})$ and low 

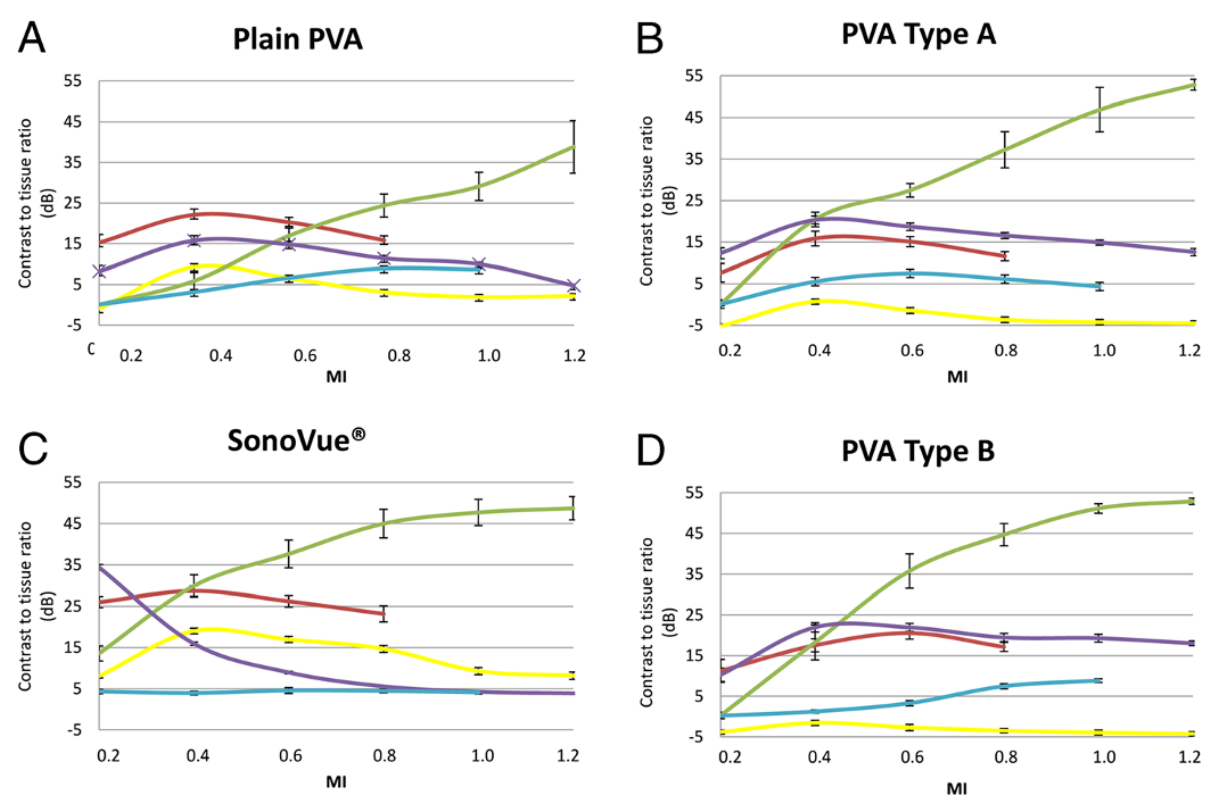

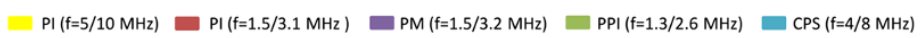

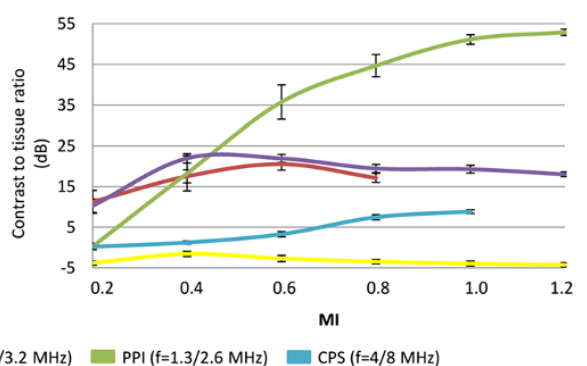

Figure 4 Contrast to tissue ratio as a function of mechanical index (MI). Contrast to tissue ratio $(\mathrm{dB})(n=38)$ as a function of $M I$ for the different contrast agents (CAs) (Plain polyvinyl alcohol (PVA) (A); PVA Type A (B); PVA Type B (C); SonoVue ${ }^{\circledast}$ (D)) and contrast sequences (pulse inversion, $\mathrm{f}=5 / 10 \mathrm{MHz}$ (yellow); pulse inversion, $\mathrm{f}=1.5 / 3.1 \mathrm{MHz}$ (red); power modulation, $\mathrm{f}=1.5 / 3.2 \mathrm{MHz}$ (purple); power pulse inversion, $\mathrm{f}=1.3 / 2.6 \mathrm{MHz}$ (green); contrast pulse sequence, $\mathrm{f}=4 / 8 \mathrm{MHz}$ (blue) included in the study. The concentration of the CAs was $10^{5}$ microbubbles $/ \mathrm{ml}$.

frequency transducer $(\mathrm{f}=1.5 / 3.1 \mathrm{MHz})$, the higher CTRvalues being obtained with the low frequency transducer (compare Figure 6A and 6B).

\section{Acoustic shadowing}

At a concentration of $10^{6} \mathrm{MBs} / \mathrm{ml}$, significant acoustic shadowing was observed for all three polymer-shelled CAs and contrast sequences (see Table 3). For concentrations lower than $10^{5} \mathrm{MBs} / \mathrm{ml}$, significant acoustic shadowing was only observed when using PPI.

\section{Discussion}

The CTR-values from the different CAs was strongly dependent on the contrast sequence and MI applied. As in previous studies $[5,10,13]$ (Poehlmann et al.: On the interplay of structural, mechanical and acoustic behaviour of multifunctional magnetic microbubbles, submitted), variations in acoustic response for the polymer-shelled CAs were observed, thus suggesting that surface modification has an impact on the acoustic properties.

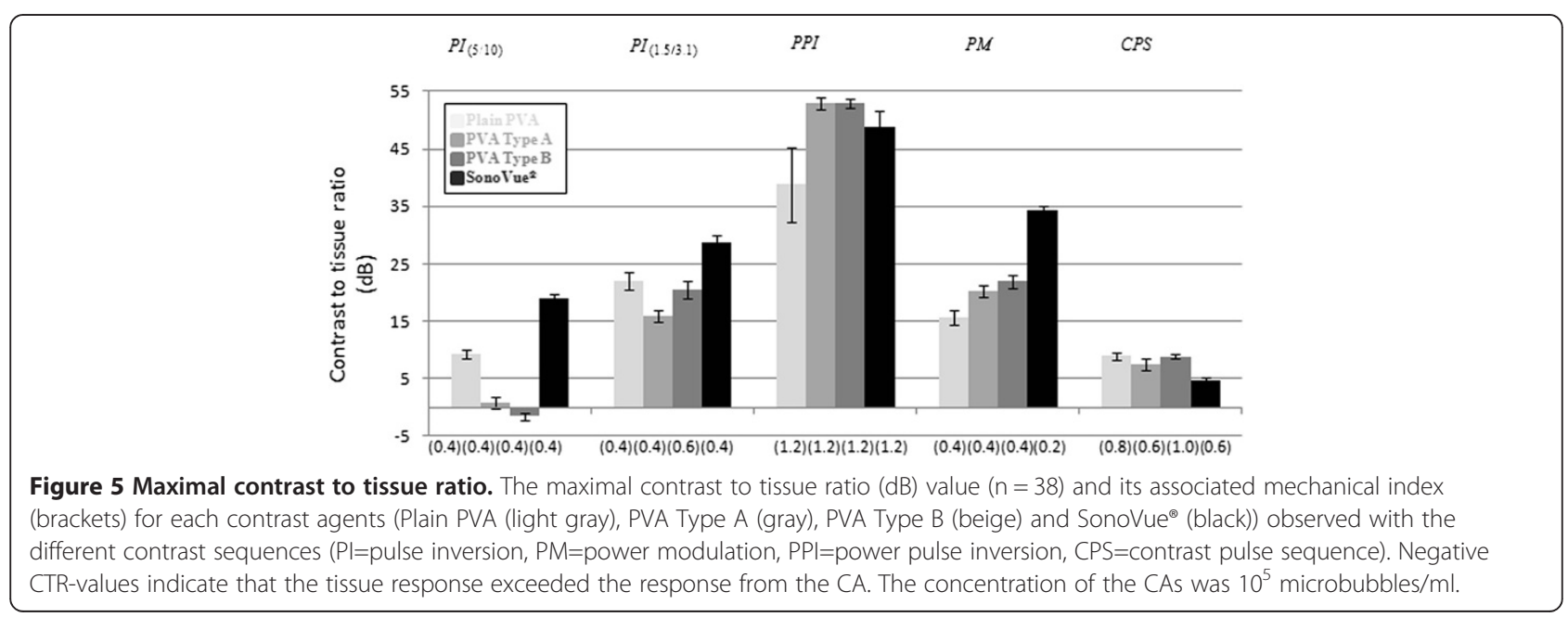




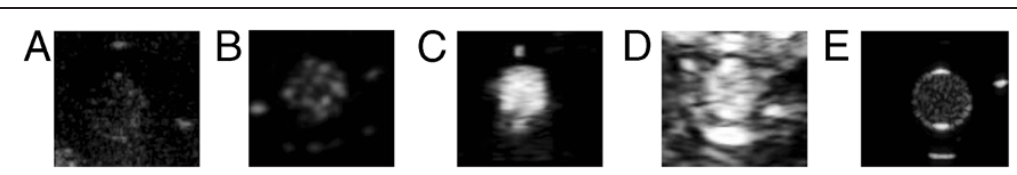

Figure 6 Ultrasound images of the tissue mimicking flow phantom containing Plain PVA. The images were obtained at maximum contrast to tissue ratio for each contrast sequences: $\mathbf{A})$ Pulse inversion ( $f=5 / 10 \mathrm{MHz}, \mathrm{Ml}=0.4)$, B) Pulse inversion $(\mathrm{f}=1.5 / 3.1 \mathrm{MHz}, \mathrm{Ml}=0.4)$,

C) Power pulse inversion ( $f=1.3 / 2.6 \mathrm{MHz}, \mathrm{Ml}=1.2)$, $\mathrm{D})$ Power modulation ( $\mathrm{f}=1.5 / 3.2 \mathrm{MHz}, \mathrm{Ml}=0.4)$, $\mathbf{E})$ Contrast pulse sequence $(f=4 \mathrm{MHZ}, \mathrm{Ml}=$ 1.0). The concentration of the CAs was $10^{5}$ microbubbles $/ \mathrm{ml}$.

Overall, the PPI generated the highest CTR-values for all CAs included in the study. PPI is a decorrelation detection contrast sequence with the ability to effectively cancel the fundamental signal and enhance the harmonic signals due to the transmission and detection of three pulses for each echo line [20]. Both the oscillation and the destruction of CAs between the pulses, results in a high degree dissimilarities of the harmonic components, especially at high pressure, i.e. at higher MI. However, not only $\mathrm{CA}$, but tissue also generates a nonlinear response when exposed to higher MI. Therefore, in order to obtain an image with minimal tissue influence, the received signal is filtered between the first and the second harmonic peak for the PPI contrast sequence. The main reason for this is that the harmonic signals from the CA have a wider bandwidth than those from the surrounding tissues [18]. The use of flexible and easily destroyed CA would result in a high decorrelation of the returning ultrasound pulses, which appears to be optimal for PPI sequence. This fact is confirmed by the high CTR-values for the thin-shelled SonoVue ${ }^{\circ}$ using PPI. However, the thick polymer-shelled CAs used in this study produced CTR-values of the same magnitude as SonoVue, even though the thickshelled CAs fracture at higher MIs and this process is different compared to thin-shelled MBs [11]. The strong acoustic signal from the polymer-shelled CAs using PPI may be a result of shell defect caused by the ultrasound exposure resulting in release of free gas [18].
The ability to generate harmonics is highly dependent on the visco-elastic properties of the shell, which are determined by the shell thickness and its composition [21]. The polymer-shelled CAs have a stiffer and thicker shell than SonoVue ${ }^{\circ}$, implying that a higher MI is needed to obtain a sufficient nonlinear response from these CAs $[23,24]$. But, in the case for PM and PI, a higher MI generates too much non-linear tissue signal to obtain high CTR-values. The difference in shell properties appears therefore to be the main reason for the fact that PI and PM perform better with SonoVue ${ }^{\circ}$ than with the polymeric shelled CAs.

The two tested contrast sequences based on PI (low frequency $f=1.5 / 3.1 \mathrm{MHz}$ and high frequency $\mathrm{f}=5 / 10 \mathrm{MHz}$ ) generated different acoustic response, the lower frequency being superior for all CAs tested. The flexible shell of SonoVue allows for large radius excursions giving rise to a well-defined resonance frequency [25]. This resonance frequency corresponds to the frequency range of the low frequency transducer, which in fact explains the higher CTR-values in this case. The polymer-shelled CAs are stiffer, which results in a considerable damping and a higher and less well-defined resonance frequency. Nevertheless, the low frequency transducer generated the highest CTR-values also with these CAs. One probable explanation to the observed phenomena, is that the high frequency signal results in higher pressure amplitudes at a certain MI, which cause a higher nonlinear response from tissue and hence, a decreased CTR-value. The frequencydependant progressive distortion of the wave as it travels

Table 3 Acoustic shadowing

\begin{tabular}{|c|c|c|c|c|c|c|c|c|c|c|c|c|c|c|c|}
\hline \multirow[t]{3}{*}{ CA } & \multicolumn{3}{|l|}{ PI } & \multicolumn{3}{|l|}{ PI } & \multicolumn{3}{|l|}{ PM } & \multicolumn{3}{|l|}{ PPI } & \multicolumn{3}{|l|}{ CPS } \\
\hline & \multicolumn{3}{|c|}{$(f=5 / 10 \mathrm{MHZ})$} & \multicolumn{3}{|c|}{$(f=1.5 / 3.1 \mathrm{MHz})$} & \multicolumn{3}{|c|}{$(f=1.5 / 3.2 \mathrm{MHz})$} & \multicolumn{3}{|c|}{$(\mathrm{f}=1.3 / 2.6 \mathrm{MHz})$} & \multicolumn{3}{|c|}{$(f=4 \mathrm{MHz})$} \\
\hline & $10^{6}$ & $10^{5}$ & $10^{4}$ & $10^{6}$ & $10^{5}$ & $10^{4}$ & $10^{6}$ & $10^{5}$ & $10^{4}$ & $10^{6}$ & $10^{5}$ & $10^{4}$ & $10^{6}$ & $10^{5}$ & $10^{4}$ \\
\hline \multirow[t]{2}{*}{ Plain PVA } & $p<0.001$ & NS & NS & $p<0.001$ & NS & NS & $p<0.001$ & NS & NS & $p<0.001$ & $p<0.01$ & NS & $p<0.001$ & NS & NS \\
\hline & $\overline{(0.4)}$ & $(0.4)$ & $(0.4)$ & $(0.4)$ & $(0.4)$ & $(0.4)$ & $(0.4)$ & (0.4) & (0.4) & $(1.2)$ & $(1.2)$ & $(1.2)$ & $(1.0)$ & $(0.8)$ & $\overline{(0.8)}$ \\
\hline \multirow[t]{2}{*}{ PVA Type A } & $p<0.001$ & NS & NS & $p<0.001$ & $p<0.001$ & NS & $p<0.001$ & $p<0.001$ & NS & $p<0.001$ & $p<0.001$ & $p<0.001$ & $p<0.001$ & NS & NS \\
\hline & $(0.4)$ & $(0.4)$ & $(0.4)$ & $(0.4)$ & $(0.4)$ & $(0.4)$ & $(0.4)$ & $(0.4)$ & $(0.6)$ & $(1.2)$ & $(1.2)$ & $(1.2)$ & $(0.8)$ & $(0.6)$ & (0.6) \\
\hline \multirow[t]{2}{*}{ PVA Type B } & $p<0.001$ & NS & NS & $p<0.001$ & NS & NS & $P=0.01$ & NS & NS & $p<0.001$ & $p<0.001$ & $p<0.05$ & $p<0.001$ & NS & NS \\
\hline & $(0.4)$ & $(0.4)$ & $(0.4)$ & $(0.4)$ & (0.6) & $(0.4)$ & $(0.6)$ & $(0.4)$ & (0.6) & $(1.2)$ & $(1.2)$ & $(1.2)$ & $(1.0)$ & $(1.0)$ & $(1.0$ \\
\hline
\end{tabular}

Observations of acoustic shadowing, at the maximal contrast to tissue ratio (dB) value and its associated mechanical index (brackets) for the different types of the polymer-shelled shelled CA at three concentrations; $10^{6} \mathrm{MBs} / \mathrm{ml}, 10^{5} \mathrm{MBs} / \mathrm{ml}, 10^{4} \mathrm{MBs} / \mathrm{ml}$. A significant shadowing effect is marked with its $\mathrm{p}$-value and a non significant shadowing effect with NS (not significant).

$\mathrm{PI}=$ Pulse Inversion, PM = Power modulation, $\mathrm{PPI}=$ power pulse inversion, $\mathrm{CPS}=$ Contrast pulse sequence, PVA = Polyvinyl alcohol, NS = not significant. 
distally is an additional explanation. This influence was, however, reduced in the present experimental setup by the different imaging depths for the low and high frequency transducers.

The penetration of the ultrasound beam at a high concentration of $\mathrm{MBs}$ is reduced due to scattering and absorption [26]. It has been shown that acoustic shadowing is less pronounced at concentrations where there is a linear relationship between the concentration of CA and backscattered intensity [27]. In the present study, acoustic shadowing was observed for concentrations of $10^{6} \mathrm{MBs} / \mathrm{ml}$, irrespective of the CA and contrast sequence used, indicating a non-linear relation between $\mathrm{CA}$ concentration and image intensity at this concentration. Acoustic shadowing was most pronounced for PPI, where significant shadowing was observed even at low CA concentrations. This can, at least partly, be explained by the high pressure amplitudes for this contrast sequence, which in other studies have shown to highly increase the attenuation in a contrast media suspension [28-30]. These findings are contradictory to the observation for CPS, where acoustic shadowing was not observed for the lower CA concentrations of the polymeric CA even though the pressure amplitude and the frequency was higher than for PPI. However, it should be mentioned in this context that the attenuation also has shown to be dependent on the transducer design and incident frequency spectra [28]. These results were found in a single element transducer setting but related findings was also obtained in a study utilizing clinical ultrasound systems [31]. In that study, machine-specific pre-and post processing algorithms were pointed out as an explanation for variation in the relationship between CA concentration and video intensity between ultrasound systems. In the current performed study, broadband transducers were used and the impact of the transducer design in combination with other machine-specific factors remains to be defined.

The in-vitro flow phantom setup offers standardized conditions for image acquisition. However, some limitations must be taken into account. It would have been optimal to use one single ultrasound system equipped with different contrast sequences over a wide frequency range. This was not possible since the clinical ultrasound systems available on the market today are limited to a few specific frequencies and contrast sequences. Still, this study provides an indication of which settings to use in order to visualize the polymeric CAs. A closed system was employed in the present study, implying that concentration loss due to $\mathrm{MB}$ destruction might have influenced the obtained CTRvalues. However, the short ultrasound exposure time in combination with a relatively large volume of the CA solution, limited the effect of any MB destruction. Furthermore, the image acquisition was also performed in a randomized order in order to minimize possible influence of $\mathrm{MB}$ destruction. Different frame rates were used in the study. As a consequence, the acquisition time differed between the ultrasound systems tested. However, variations within the stored image sequences were limited as a homogenous CA mixture was used. In addition, a relatively high flow was applied which minimize the effect of eventual MB destruction in the field of view. Furthermore, if air bubbles were observed, a new image sequence was obtained. MBs motion is known to influence multi-pulse techniques using phase modulations. Although it has been demonstrated that the influence of axial motion of the MBs is much higher than that of lateral motion [32], the lateral motion of the $\mathrm{MBs}$ in our experimental setting might have influenced the tested contrast sequences. The present experimental setup mimicked the in-vivo imaging situation in the macrocirculation, in which contrast enhanced imaging can be used for detection of various clinical scenarios, e.g. left ventricle opacification, endocardial border delineation and detection of atherosclerotic plaques. Nevertheless, in order to fully evaluate the potential of the polymer-shelled CAs, future studies need to focus on the ability to visualize the microcirculation and to quantify tissue perfusion.

The polymer-shelled CAs has high mechanical and chemical stability [8], facilitating a possible use of the MBs as a carrier for different substances that can be incorporated into the shell or attached to the shell surface. It has thereby the potential to be of value in new clinical applications as, for example, multimodality imaging, targeted imaging and drug delivery. In particular, multimodal imaging where ultrasound and MRI are combined can add incremental value since these imaging methods are complementary in the diagnostic practice. The results of this study provide a clear indication of the optimal settings and contrast sequences for the polymershelled CAs, however, in-vivo imaging will certainly be more challenging and factors such as injection speed and concentration focus position and gain levels remain to be optimized. There is therefore a need for new invitro and in-vivo studies in order to further evaluate the applicability of the developed CAs, especially in the evaluation of microcirculation and tissue perfusion.

\section{Conclusions}

The present results demonstrate that the three polymershelled CAs have the potential to enhance ultrasound images when using commercially available contrast sequences, the highest CTR-values being obtained with PPI at higher MI. Surface modification seems to have an impact on the acoustic properties, as illustrated by the differences in CTR-values and acoustic shadowing between the three polymer-shelled CAs currently tested. A significant acoustical shadowing observed with all contrast 
sequences at a CA concentration of $10^{6} \mathrm{MBs} / \mathrm{ml}$ implies that this $\mathrm{CA}$ concentration is too high for a linear relation between $\mathrm{MB}$ concentration and image intensity.

\section{Competing interests}

The authors declare that they have no competing interests.

\section{Authors' contributions}

$M L, L A ̊ B, A B$ participated in the initiation and design of the study. $L O, S M$ and GP produced the polymer-shelled contrast agent used in the study. $\mathrm{ML}, \mathrm{ML}, \mathrm{JN}, \mathrm{KC}$ and $\mathrm{AB}$ participated in data collection and analysis of the results. All authors read and approved the final manuscript.

\section{Acknowledgements}

This study was supported by the European commission project 3MiCRON (245572), within the frame of the seventh framework program.

\section{Author details}

'Department of Medical Engineering, School of Technology and Health, KTH Royal Institute of Technology, Alfred Nobels Allé 10, 141 52, Huddinge, Stockholm, Sweden. ${ }^{2}$ Department of Molecular Medicine and Surgery, Karolinska Institutet, Solna, Sweden. ${ }^{3}$ Department of Chemical Sciences and Technologies, Università di Roma Tor Vergata, Rome, Italy. ${ }^{4}$ Division of Clinical Physiology, Karolinska University Hospital, Huddinge, Sweden.

Received: 25 June 2013 Accepted: 27 August 2013

Published: 29 August 2013

\section{References}

1. Gramiak R, Shah PM: Echocardiography of the aortic root. Invest Radiol 1968, 3:356-366.

2. Feinstein SB, Shah PM, Bing RJ, Meerbaum S, Corday E, Chang BL, Santillan G, Fujibayashi Y: Microbubble dynamics visualized in the intact capillary circulation. J Am Coll Cardiol 1984, 4:595-600.

3. Sboros $\mathrm{V}$, Tang MX: The assessment of microvascular flow and tissue perfusion using ultrasound imaging. Proc Inst Mech Eng H 2010, 224:273-290.

4. Senior R, Andersson O, Caidahl K, Carlens P, Herregods MC, Jenni R, Kenny A, Melcher A, Svedenhag J, Vanoverschelde IL, et al: Enhanced left ventricular endocardial border delineation with an intravenous injection of SonoVue, a new echocardiographic contrast agent: a European multicenter study. Echocardiogr 2000, 17:705-711.

5. Brismar TB, Grishenkov D, Gustafsson B, Harmark J, Barrefelt A, Kothapalli SV, Margheritelli S, Oddo L, Caidahl K, Hebert H, Paradossi G: Magnetite nanoparticles can be coupled with microbubbles to support multimodal imaging. Biomacromolecules 2012, 13:1390-1399.

6. Paradossi G, Cavalieri F, Chiessi E, Ponassi V, Martorana V: Tailoring of physical and chemical properties of macro- and microhydrogels based on telechelic PVA. Biomacromolecules 2002, 3:1255-1262.

7. Cavalieri F, El Hamassi A, Chiessi E, Paradossi G: Stable polymeric microballoons as multifunctional device for biomedical uses: synthesis and characterization. Langmuir 2005, 21:8758-8764.

8. Cavalieri F, El Hamassi A, Chiessi E, Paradossi G, Villa R, Zaffaroni N: Tethering functional ligands onto shell of ultrasound active polymeric microbubbles. Biomacromolecules 2006, 7:604-611.

9. Talu E, Hettiarachchi K, Zhao S, Powell RL, Lee AP, Longo ML, Dayton PA: Tailoring the size distribution of ultrasound contrast agents: possible method for improving sensitivity in molecular imaging. Mol Imaging 2007, 6:384-392.

10. Grishenkov D, Pecorari C, Brismar TB, Paradossi G: Characterization of acoustic properties of PVA-shelled ultrasound contrast agents: linear properties (part I). Ultrasound Med Biol 2009, 35:1127-1138.

11. Grishenkov D, Pecorari C, Brismar TB, Paradossi G: Characterization of acoustic properties of PVA-shelled ultrasound contrast agents: ultrasound-induced fracture (part II). Ultrasound Med Biol 2009, 35:1139-1147.

12. Sciallero C, Paradossi G, Trucco A: A preliminary in vitro assessment of polymer-shelled microbubbles in contrast-enhanced ultrasound imaging. Ultrasonics 2012, 52:456-464.

13. Sciallero C, Trucco A: Ultrasound assessment of polymer-shelled magnetic microbubbles used as dual contrast agents. J Acoust Soc Am 2013, 133:EL478-484.
14. Madsen EL, Zagzebski JA, Banjavie RA, Jutila RE: Tissue mimicking materials for ultrasound phantoms. Med Phys 1978, 5:391-394.

15. Jea F: Estimation of polyvinyl alcohol cryogel mechanical properties with four ultrasound elastography methods and comparison with gold standard testings. IEEE Trans Ultrason Ferroelectr Frea Control 2007, 54:498-509.

16. Dähne L, Egri G: Preparation of magnetic microbubbles with PVA wall and use of them. German patent, DE. 102011000 264.2-43.

17. Schneider M: Characteristics of SonoVuetrade mark. Echocardiogr 1999, $16: 743-746$

18. Kaufmann BA, Wei K, Lindner JR: Contrast Echocardiography. Curr Probl Cardiol 2007, 32:51-96.

19. Phillips $P$, Gardner E: Contrast-agent detection and quantification. Eur Radiol 2004, 14(Suppl 8):P4-10.

20. Averkiou M, Powers J, Skyba D, Bruce M, Jensen S: Ultrasound contrast imaging research. Ultrasound Q 2003, 19:27-37.

21. Leong-Poi H, Song J, Rim SJ, Christiansen J, Kaul S, Lindner JR: Influence of microbubble shell properties on ultrasound signal: implications for lowpower perfusion imaging. J Am Soc Echocardiogr 2002, 15:1269-1276.

22. Tiemann K, Schlosser T, Pohl C, Bimmel D, Wietasch G, Hoeft A, Likungu J, Vahlhaus C, Kuntz S, Nanda NC, et al: Are microbubbles free flowing tracers through the myocardium? Comparison of indicator-dilution curves obtained from dye dilution and echo contrast using harmonic power Doppler imaging. Echocardiogr 2000, 17:17-27.

23. Cavalieri F, Flnelli I, Tortora M, Mozetic P, Chiessi E, Polizio F, Brismar TB, Paradossi G: Polymer micobubbles as diagnostic and therapeutic Gas delivery device. Chem Mater 2008, 20:3254-3258.

24. Hoff L: Acoustic Characterization of Contrast Agents for Medical Ultrasound Imaging. AA Dordrecht: Kluwer Academic Publisher; 2001.

25. Greis C: Technology overview: SonoVue (Bracco, Milan). Eur Radiol 2004, 14(Suppl 8):P11-15.

26. de Jong N, Hoff L, Skotland T, Bom N: Absorption and scatter of encapsulated gas filled microspheres: theoretical considerations and some measurements. Ultrasonics 1992, 30:95-103.

27. Lampaskis M, Averkiou M: Investigation of the relationship of nonlinear backscattered ultrasound intensity with microbubble concentration at low MI. Ultrasound Med Biol 2010, 36:306-312.

28. Chen $\mathrm{Q}$, Zagzebski J, Wilson T, Stiles T: Pressure-dependent attenuation in ultrasound contrast agents. Ultrasound Med Biol 2002, 28:1041-1051.

29. Stride $\mathrm{E}$, Saffari $\mathrm{N}$ : Investigating the significance of multiple scattering in ultrasound contrast agent particle populations. IEEE Trans UItrason Ferroelectr Frea Control 2005, 52:2332-2345.

30. Tang MX, Eckersley RJ: Frequency and pressure dependent attenuation and scattering by microbubbles. Ultrasound Med Biol 2007, 33:164-168.

31. Jayaweera AR, Skyba DM, Kaul S: Technical factors that influence the determination of microbubble transit rate during contrast echocardiography. J Am Soc Echocardiogr 1995, 8:198-206.

32. Fanglue Lin CC, Riccardo M, Jacopo V, François V, Francesco G, Olivier B: Influences of bubble motion to second-harmonic inversion imaging. In Proceedings of the 2012 IEEE Ultrasonics symposium: 7-10 October 2012. Dresden:675-678.

\section{doi:10.1186/1476-7120-11-33}

Cite this article as: Larsson et al:: Visualization of multimodal polymershelled contrast agents using ultrasound contrast sequences: an experimental study in a tissue mimicking flow phantom. Cardiovascular Ultrasound 2013 11:33. 